\title{
Dealing with Wandering in Institutional Care: Exploring the Field
}

\author{
Claudia Müller \\ International Institute for Socio-Informatics (IISI) \\ and University of Siegen \\ 53111 Bonn, Germany \\ claudia.mueller@uni-siegen.de
}

\author{
Lin Wan, Volker Wulf \\ University of Siegen \\ Institute for Information Systems, \\ 57076 Siegen, Germany \\ \{lin.wan; volker.wulf\}@uni-siegen.de
}

\begin{abstract}
We will present a case study on institutional care workers' strategies in dealing with wandering dementia patients. We will highlight their day-to-day practices, permanently balancing conflicting demands around keeping harm away from residents with wandering behavior. The risk of harm is multifactorial and also socially, culturally and organizationally defined. A closer examination of how the staff uses non-tech, lowtech and information and communication technology (ICT) artifacts in their daily practice helps us gain a better understanding of their practical problems and rationales for further ICT design.
\end{abstract}

Keywords-dementia care; wandering management; institutional care

\section{INTRODUCTION}

This case study focuses on the strategies the institutional care workers use on a daily basis when dealing with the wandering behaviors of dementia patients, and how they have adapted to the situation and finally developed these strategies. Wandering behavior, which is common in people with dementia, is sometimes regarded as beneficial, as it is often the last way to express oneself when the disease has progressed quite far. On the other hand, it is likely to cause physical harm, emotional distress and death [1]. The caregiving for dementia patients with wandering behavior is often an overwhelming burden and a demanding challenge for the institutional caregivers. When caring for people with dementia, it always comes to a situation of collision and excess of autonomy bounds, which arouses controversy around privacy concerns.

While there are guidelines and standards of care, as well as legal basis and in-house standards, there is still much uncertainty when they are actually used in daily practice. As Lucy Suchman has pointed out, people's behavior is "highly dependent upon its material and social circumstances, focusing on moment-by-moment interactions between actors, and between actors and the environments of their action” [2]. This suggests that when designing technology for the field, it is necessary to carefully examine the actual situations, how all the actors act in practice, and how situational and organizational factors fit into the cognitive process.

This work is framed under a multidisciplinary research project aiming at designing a passive monitoring appliance for wandering management, in order to lighten the caregiver's burden as well as preserve as much of the patient's autonomy as possible. This paper describes our first findings in understanding the field.

\section{STATE OF THE ART}

Most studies on assistive technologies for the demented and/or their caregivers stress the massive value tensions that need to be considered in the research and development process $[3,4]$. That's why some approaches aim at grasping a holistic knowledge upon attitudes, fears and expectations of familial or professional caregivers - and when possible of demented persons themselves - towards ICT adoption and usage, such as GPS locating systems [1, 5, 6]. Others focus on actual usage and reflections of ICT devices and their respective functionalities $[4,7]$ in their research.

While a branch of research on managing wandering behavior measures in long-term facilities, focuses on "non- or low-tech" measures in different categories, such as medication, environmental manipulation (e.g. camouflage of doors), or activity programs [8]. Other research works tend to support the field with more technical intervention [4].

While there are several research works on new ICT developments for the field, we believe there is still a lack of profound understanding of the current practices of wandering management. And more importantly, what are the intrinsic drive and hesitation of the facilities to create a specific strategy? Not only ICT artifacts incorporate value trade-offs, many other perspectives (e.g. ethical, legal et al.) are weighed against each other to finally consolidate a certain practice. We are especially motivated to verify these perspectives in a study that encompasses different measures and strategies used by care workers from non-, low- to high-tech solutions. By this we are trying to gain a deeper insight into the practical problems of care workers and the strategies they use to balance different value demands in their every-day work.

\section{METHODOLOGICAL APPROACH}

We have conducted 15 semi-structured interviews and 17 hours of participant observation in three different institutional settings; in care homes, a geriatric ward in a hospital and in assisted living arrangements for people with dementia. Professional caregivers were care workers on wards, who work 
directly with those suffering from dementia and also with others in management positions. In addition, we use information of three interviews with relatives of institutionalized persons with dementia, two of them adult children and one wife.

The interviews, lasted from 1,5 to 2 hours, were recorded and transcribed. Field notes were taken during the participant observations. Data analysis largely followed the grounded theory methodology [12] and an analysis was conducted in several steps. We started with open coding, describing loose categories that came up in the interviews. Subsequently, the codes were then organized into higher-level categories. Some of the results of this process are the topics we will describe in this paper.

\section{UNDERSTANDING THE FIELD}

\section{A. Constructional Measures}

When new dementia care facilities are constructed, constructional measures are taken into consideration as much as possible. In Germany, the entrance for such "open wards" has to be open at all times according to legal regulations. This creates a great challenge for the organization, having to guarantee for the safety of patients with wandering tendencies. During the construction, many doors are intentionally installed in a way that requires much more effort to open them than normal doors. Some doors use opaque material, such as frosted glass, to avoid activities from outside "attracting" the patients inside.

Subsequently, the wards usually camouflage the exits and areas to which wandering patients should not have access. The exits or doors to the fire escapes, which are not to be locked, are sometimes covered with curtains. In one facility they have placed a screen in front of the door to hide it. These measures have received extremely mixed acceptance from the personnel. Supporters consider these measures to be a legitimate expedience without directly restricting the autonomy of the patients. Opposing opinions deem these measures as a detriment to human dignity, as the camouflage creates a false reality and takes advantage of a user group who are not able to tell the difference.

Creating "endless hallways" is another commonly used measure, which was found in a flat shared by sufferers of dementia that we visited. The hallway is built in a circular form, around a shared activity area in the middle. This lets the patients to take walks through the hallway, but they never reach the exit. However, some interviewees criticized this new constructional principle. They called this measure inhuman, as the patients never see the beginning or the end of the hallway and can then be further confused.

\section{B. Technical Installations}

\section{1) Video Camera}

In one facility a video camera was installed on the ceiling of the lounge, which is also used as the dining room for the residents. Although the camera was installed when the building was newly constructed several months ago, it has not been used yet. The ambiguous legal issues need to be clarified before the camera can be put to use. During the past few months the facility management has been researching and negotiating with lawyers and the general staff do not expect a fast solution to this situation. In another nursing home with an affiliated dementia ward, video cameras are installed in the main entrance. The reasoning behind the installation is to provide more safety to the residence from unwanted strangers. As an open ward, there are no fixed visiting times; the relatives of the residents can visit the ward at any time in the day. This openness increases the worries and concerns of the residents about their safety, because suspicious looking strangers have been spotted in front of the main entrance several times. Since then the cameras have been installed, but the residents are still anxious. After receiving the consent of the inhabitants, additionally, the front door is now locked during the night. Feeling like they are doing something illegal, the manager refers to the agreement with the residents and that "all elderly people would lock their front door at home in the night".

\section{2) Door Safety System}

Door safety systems can be found in many stationary facilities. We encountered several problems and uncertainties in knowledge of how these doors work.

In the dementia ward of an old people's home, a sensor system was installed at the main entrance. Five residents wear a special wristband, when they pass the front door an alarm will show on the ward's phone. However, the sensor is not connected to an alarm system. When we asked whether the ward needed a technical upgrade, the answer was no: "if you wire up the whole house, it will be like a prison". The major problem, as mentioned by the doorman, was the reliability on the system and its performance. It has happened more than once that the alarm was not triggered even though someone has passed through the front door. Another problem is the battery life: there is no battery indicator on the wristband; the only sign that it needs to be recharged is when it stops working. And the battery only lasts for 3 months if used by active residents, which is much shorter than what the director expected (2 years). Furthermore, the caregiving staff are facing a pragmatic problem: when an alarm appears on the phone display, it will only show “door open”, but does not display the name of the resident. From the alarm there is no clue who has left the ward, e.g. if a "fast" or a "slow" walker has left. This is not a situation caused by technical difficulties, but rather is due to the manager's decision. He believes that making names transparent will damage the autonomy of the patients, thus an official certificate should be needed.

In a flat shared by dementia patients a specially designed door lock system is installed. To open the door one has to rotate the door nob and press a green button on the wall at the same time to keep an alarm from being triggered. From different care workers we got different answers as to which buttons have to be pushed to open the door. In the end we learned that this system 
was not activated at all and the staff was not aware of this. A large old people's home embedded chips into the residents' shoes. These chips are supposed to trigger an alarm when the residents go through certain exits. These chips are unobtrusive for the residents, as they are hidden in the shoes. One decisive factor for using them is that the caregivers have to make sure that the inhabitants have their shoes on. The caregiving staff has developed according routines to constantly check whether residents are wearing their shoes. Another factor for using the chips is that the system only gives an alert when the patient walks through the official doors and but it does not give an alert when emergency exits are used, which has happened several times.

\section{3) Sensor Mat}

In one facility a sensor mat is put in front of beds of patients with a risk of falling. This is used to inform the nurses that the patient has left the bed, especially in the night, so that the nurses can take care of them accordingly. We have discussed this topic with the manager of another facility, where we received a totally opposite attitude towards the sensor mats. The manager perceived the patient's room to be his/her only private space in the facility. The patients are constantly being watched when they leave their rooms, so he felt that they should have full freedom in their own rooms. Although we don't have explicit utterances from relatives about the use of sensor mats, we see a tendency of relatives to value safety measures over privacy concerns. This is often grounded in the overwhelming anxiety and excessive demands relatives feel when caring for their demented spouses/parents at home and which in many cases leads to the patients' transfer to a care home.

\section{4) GPS Location Systems}

In one facility the manager told the relatives to look for a "closed facility" as the care workers were not able to manage his heavy wandering behavior. As the sons and wife wanted him to stay, they proposed using a GPS locating system. The manager agreed on the basis that the family would be in charge of operating the system. In general, most facilities are reluctant to use such systems, as they fear litigation problems due to privacy issues: "We do not know if this would be seen as a freedom-depriving measure".

\section{Human Strategies}

1) Patient Photo for Searches

Normally, when a patient enters a facility for the first time a photo will be taken as a management requirement. In general, these photos have helped caregivers look for the patients that have wandered off. But as a photo is a privacy sensitive medium, how each facility handles patient photos varies considerably. In some facilities photos are a part of the patient record. The patient record can be printed out or sent by email to other caregivers so that they can use the photo to locate the wandering patient. One facility uses photos very cautiously, because of privacy concerns. Here the photos are only saved on the camera itself.

How much the doorman is allowed to access the patients' photos is also not the same among the interviewed facilities. On some wards it's standard measure to send a photo of the wandering patient to the doorman, so that he can easily recognize the patient if he passes by. But on wards where photos are only stored on the camera, it's forbidden to send a photo to the doorman because of privacy concerns.

2) Continuous Observation and Assessment

In many facilities, the caregivers said that they have to constantly keep an eye on the patients' activities and assess the situation. One geriatric hospital ward has a high fluctuation among hospitalized patients and some patient's wandering tendency are not yet known when they arrive. "Most of the time we don't know whether they have a wandering tendency. Sometimes we can predict it based on the patient's behaviors. For example when they put their coat on or take off their pajamas and put on outdoor clothes, or when they pack a suitcase and move towards the main exit. When this happens, we have to grab them and ask what their plan is."

In other "not so busy" facilities, the caregivers still face the big challenge of staying alert to patient's movements in the ward, especially when they are doing care routines in a room. Due to this, the doors are normally kept open, so that they can hear the movements in the hallway. In one facility, the nurses put a barrier in the hallway when they have to enter a patient room. If a patient wants to pass through, he has to move these barriers and the nurses will hear the noise and know what's happening in the hall. Commonly used barriers are e.g. the nursing cart used during the morning and evening rounds, or toilet chairs. This is a compromise for the nurses that they see somewhat ambiguously, because normally they try to have the floors empty so that the residents can walk safely. But they do not know how to solve this practical problem otherwise due to the low number of care workers in one shift.

\section{3) Activity Intervention}

For dementia patients, an important intrinsic driving force for wandering is the agitation and the urge to move. Accordingly, caregivers have developed measures to intentionally keep patients busy and distracted. Activities such as reading, chatting with each other, eating together, and going for walks together are the most common ones. In one facility they regularly dance together. Keeping the patients busy during the day also helps them sleep better at night, which reduces the danger of patients wandering off during the night.

4) Cross-area / Cross-ward Cooperation

In facilities with multiple wards or wards where the functions are separated into areas, patients with wandering tendencies are normally monitored 
collaboratively by care personnel from all wards/areas. "Normally we call the neighbor stations: ' $X X$ will come to you shortly, can you help bring her back?' This is how it works." "Here the care personnel from the ward opposite ours have always helped keep an eye on our patients. Everyone takes care, also the colleagues from other stations. Our two wanderers always take a walk between the wards, so everyone is involved in the monitoring."

\section{5) Cooperation with Relatives}

The caregivers sometimes call the relatives for help when they feel a strong agitation or wandering urge from the patient, or when the patient has got lost. Talking to a relative on the phone usually helps the patient calm down. Relatives are also willing to involve themselves in the care of the patient when they visit the facility. Sometimes relatives expect the institution to guarantee $100 \%$ safety and that they prevent the resident from leaving the house. These strong safety concerns often stem from the relatives' former experience of taking care of the demented at home and its excessive demands. This wish is in principle against the philosophy of patient-centered care, which supports wandering as a meaningful and soothing activity from the manager's point of view. But such philosophy often puts a lot of pressure on the nurses who have to constantly balance principles of patient-centered care and risk management (to prevent harm) in their daily work with the residents.

\section{DISCUSSION}

Dealing with wandering in dementia for care facilities is a challenging task. Care workers have to continuously look for a balance between their own and the patients' risks. Their personal risk is in possible litigation aspects when offending the residents' rights to autonomy - but they are also responsible of protecting the residents from any harm. However, litigation aspects are far from being clearly defined. That's why there are attempts to define measures as „,normal and usual", such as the heavy doors or locking the front door at night which, from another point of view, could possibly be defined as a freedom-depriving measures. Dahl et al. mention that new care culture and conceptualization of dementia, such as person-centered care [11], has been widely adopted in care facilities - but not in the public so far [4]. Actually, these new care philosophies are regarded as very important in all of the facilities in our sample. But for the care workers it is hard to implement the principles in their daily work. It becomes clear, that to a certain extent, risks are socially and also culturally constructed [1] and are highly dependent on the organizational structure.

Those who work directly with the residents have to constantly make compromises to meet all requirements set up by different stakeholders, such as management, doctors, IT administration and relatives. The findings of this study thus have several implications for methodological and design related aspects. Our findings have shown that non- and low- tech supportive measures have received controversial reviews from different stakeholders; they also indicate that assistive technologies will almost certainly be controversially perceived. This means that the design process must be deeply grounded in the respective practices of each target group, which can be different even from house to house. Practice-based and participatory design methodologies should therefore be deployed. Linked to this methodological requirement is a need to open up a common discussion sphere for all stakeholders to make the situated and individual practical problems more visible and to find better compromises that serve the practice. The situated character of practical problems also hints at the need for design approaches to allow assistive systems to adapt to individual requirements, such as end-user development. This approach would also allow for future system adaption when discourses and requirements change over time.

\section{CONCLUSION}

Our study contributes to research on assistive technology for caregivers in long-term dementia care. Examining care workers' non- and low-tech strategies helps understand their concerns towards locating technologies against the background of their every-day practical problems and their needs to balance compromises in the management of wandering in dementia.

\section{REFERENCES}

[1] L. Robinson et al., „Balancing rights and risks: Conflicting perspectives in the management of wandering in dementia“, Health, Risk \& Society, Bd. 9, Nr. 4, S. 389-406, 2007.

[2] L. A. Suchman, Plans and Situated Actions: The Problem of HumanMachine Communication. Cambridge University Press, 1987.

[3] S. Lindsay et al., „Empathy, participatory design and people with dementia“, in Proceedings of CHI 2012, New York, NY, USA, 2012, S. 521-530.

[4] Y. Dahl, K. Holbø, „Value biases of sensor-based assistive technology: case study of a GPS tracking system used in dementia care“, in Proceedings of DIS 2012, New York, NY, USA, 2012, S. 572-581.

[5] R. Landau, S. Werner, G. K. Auslander, N. Shoval, and J. Heinik, „Attitudes of Family and Professional Care-Givers towards the Use of GPS for Tracking Patients with Dementia: An Exploratory Study“, $\mathrm{Br} J$ Soc Work, Bd. 39, Nr. 4, S. 670-692, Jan. 2009.

[6] C. Mueller, L. Wan, and D. Hrg, „Dealing with wandering: a case study on caregivers' attitudes towards privacy and autonomy when reflecting the use of LBS“, in Proceedings of GROUP 2010, New York, NY, USA, 2010, S. 75-84.

[7] J. Y. Tsai, P. Kelley, P. Drielsma, L. F. Cranor, J. Hong, and N. Sadeh, „Who's viewed you?: the impact of feedback in a mobile locationsharing application“, in Proceedings of CHI 2009, New York, NY, USA, 2009, S. 2003-2012.

[8] C. K. Lai, J. H. Yeung, V. Mok, and I. Chi, „Special care units for dementia individuals with behavioural problems“, in Cochrane Database of Systematic Reviews, John Wiley \& Sons, Ltd, 1996.

[9] B. Friedman, „Value-sensitive design“, interactions, Bd. 3, Nr. 6, S. 1623, Dez. 1996.

[10] P. Wright and J. McCarthy, „Empathy and experience in HCI“, in Proceedings of CHI 2008, New York, NY, USA, 2008, S. 637-646.

[11] T. Kitwood, Dementia Reconsidered: The Person Comes First, 0002 Aufl. Open University Press, 1997.

[12] A. Strauss and J. M. Corbin, Grounded Theory in Practice. SAGE, 1997. 